\title{
Observational study of patients with gastroenteropancreatic and bronchial neuroendocrine tumors in Argentina: Results from the large database of a multidisciplinary group clinical multicenter study
}

\author{
JUAN MANUEL O'CONNOR ${ }^{1,2}$, FABIANA MARMISSOLLE ${ }^{3,4}$, CLAUDIA BESTANI ${ }^{2}$, VERONICA PESCE ${ }^{2}$, \\ SUSANA BELLI $^{1}$, ENZO DOMINICHINI ${ }^{1}$, GUILLERMO MENDEZ ${ }^{2}$, PAOLA PRICE ${ }^{2}$, NORA GIACOMI ${ }^{3}$, \\ ALEJANDRO PAIROLA ${ }^{1}$, FERNANDO SÁNCHEZ LORIA ${ }^{1}$, EDUARDO HUERTAS ${ }^{1}$, CLAUDIO MARTIN ${ }^{1,6}$, \\ KARINA PATANE $^{1,6}$, CLAUDIA POLERI ${ }^{6}$, MOISES ROSENBERG ${ }^{1,6}$, ANA CABANNE $^{2}$, MIRTA KUJARUK ${ }^{2}$, \\ ANALIA CAINO $^{3,5}$, VICTOR ZAMORA ${ }^{2}$, JAVIER MARIANI $^{1}$, MARIANO DIOCA ${ }^{7}$, PATRICIA PARMA ${ }^{7}$, \\ GUSTAVO PODESTA ${ }^{8}$, OSCAR ANDRIANI $^{8}$, GABRIEL GONDOLESI $^{9}$ and ENRIQUE ROCA ${ }^{2}$ \\ ${ }^{1}$ Department of Clinical Oncology, Institute Alexander Fleming, Buenos Aires C1426ANZ; ${ }^{2}$ Oncology Unit, \\ Gastroenterology Hospital Bonorino Udaondo, Buenos Aires C1264AAA; ${ }^{3}$ SITE, Clinical Oncology, La Plata 1900; \\ ${ }^{4}$ Medical Institute Platense, Buenos Aires 1900; ${ }^{5}$ Hospital El Cruce, Buenos Aires B1888; ${ }^{6}$ Department of \\ Clinical Oncology and Thoracic Surgery Unit, Respiratory Rehabilitation Hospital María Ferrer, Buenos Aires \\ C1272AAA; ${ }^{7}$ Department of Clinical Oncology, Institute A. Roffo, Buenos Aires C1417AAA; ${ }^{8}$ Department \\ of Hepatobiliary Pancreatic Disease, Austral University Hospital, Buenos Aires B1629AHJ; ${ }^{9}$ Oncology \\ and Hepatobiliary Pancreatic Units, Favaloro Foundation, Buenos Aires C1093AAS, Argentina
}

Received January 27, 2014; Accepted February 25, 2014

DOI: $10.3892 / \mathrm{mco} .2014 .332$

\begin{abstract}
Neuroendocrine tumors (NET) include a spectrum of malignancies arising from neuroendocrine cells throughout the body. The objective of this clinical investigation of retrospectively and prospectively collected data was to describe the prevalence, demographic data, clinical symptoms and methods of diagnosis of NET and the treatment and long-term follow-up of patients with NET. Data were provided by the participating centers and assessed for consistency by internal reviewers. All the cases were centrally evaluated (when necessary) by the pathologists in our group. The tissue samples were reviewed by hematoxylin and eosin and immunohistochemical staining techniques to confirm the diagnosis of NET. In total, 532 cases were documented: 461 gastroenteropancreatic-NET (GEP-NET) and 71 bronchial NET (BNET). All the tumors were immunohistochemically defined according to the World Health Organization (WHO) and European Neuroendocrine Tumor Society criteria. The most common initial symptoms in
\end{abstract}

Correspondence to: Dr Juan Manuel O'Connor, Department of Clinical Oncology, Institute Alexander Fleming, 1180 Crámer Ave., Buenos Aires C1426ANZ, Argentina

E-mail: juanmanuel.oconnor@gmail.com

Key words: clinical data, multicenter study, neuroendocrine tumors, observational study
GEP-NET were abdominal pain, diarrhea, bowel obstruction, flushing, gastrointestinal bleeding and weight loss. The most common tumor types were carcinoid (58.0\%), non-functional pancreatic tumor $(23.0 \%)$, metastatic NET of unknown primary $(16.0 \%)$ and functional pancreatic tumor $(3.0 \%)$. Of the BNET, $89.0 \%$ were typical and $11.0 \%$ atypical carcinoids. Of the patients with GEP-NET, 59.2\% had distant metastasis at diagnosis. The locations of the primary tumors in GEP-NET were the small bowel $(26.9 \%)$, pancreas $(25.2 \%)$, colon-rectum (12.4\%), appendix (7.6\%), stomach (6.9\%), esophagus $(2.8 \%)$, duodenum $(2.0 \%)$ and unknown primary $(16.3 \%)$. The histological subtypes based on the WHO classification were well-differentiated NET $(20.1 \%)$, well-differentiated neuroendocrine carcinomas $(66.5 \%)$ and poorly differentiated neuroendocrine carcinomas $(10.3 \%)$. Overall, $67.3 \%$ of the patients underwent surgery, $41.2 \%$ with curative intent and $26.1 \%$ for palliative purposes. The 5-year survival rates were $65.1 \%$ (95\% confidence interval, 58.0-71.4\%) in GEP-NET and $100.0 \%$ in typical carcinoid of the lung. This observational, non-interventional, longitudinal study aimed to accumulate relevant information regarding the epidemiology, clinical presentation and current practices in the treatment of NET patients in Argentina, providing insight into regional differences and patterns of care in this heterogeneous disease.

\section{Introduction}

Neuroendocrine tumors (NET) include a spectrum of malignancies arising from neuroendocrine cells throughout the 
body. These tumors secrete peptides and neuropeptides that may cause distinct clinical syndromes, such as the carcinoid syndrome. A number of carcinoid tumors are clinically silent until their late presentation with mass effect. Carcinoid tumors account for $<5.0 \%$ of all digestive tumors (1-5). According to the World Health Organization (WHO) 2010 classification (6), gastroenteropancreatic-NET (GEP-NET) are subdivided into well-differentiated NET (WDET), well-differentiated neuroendocrine carcinomas (WDEC) and poorly differentiated neuroendocrine carcinomas (PDEC), whereas the old classification indicated only three types: foregut, midgut and hindgut tumors. Although they share some common characteristics, these tumors exhibit significant differences in clinical behavior, genetic characteristics, molecular and biochemical profiles and therapeutic approach. GEP-NET are considered as fairly rare disease entities that pose several clinical challenges.

Approximately $75.0 \%$ of all NET cases manifest in the GEP system (5). Recent data from the Surveillance, Epidemiology and End Results (SEER) program in the United States, which is the largest series of NET cases reported to date with a focus on incidence, prevalence and prognosis factors, suggest that the incidence and prevalence of NET have increased significantly over the past 3 decades. The incidence of NET in the United States in 2004 was 5.09-5.42/100,000 and the prevalence was 35/100,000 individuals (7), which may be explained by the increased awareness and improved diagnostic techniques. Whether this also reflects a true increase of incidence remains unclear $(8,9)$. Unlike the increase in incidence and prevalence observed in SEER, there was no statistically significant difference in survival duration among patients with local and regional NET. The complexity, heterogeneity and rarity of NET have contributed to the slow development of therapies for this disease. GEP-NET are known for their lack of defining symptoms, which may lead to a delay in diagnosis. However, on metastasis, the patients may develop debilitating symptoms associated with the release of highly bioactive substances.

These symptoms of metastasis, referred to as the carcinoid syndrome, are associated with significant morbidity and are quite vague, frequently leading to misdiagnosis of a more common disorder, such as irritable bowel syndrome. Misdiagnosis puts carcinoid syndrome patients at higher risk for carcinoid crisis, the most immediate life-threatening complication of carcinoid syndrome. In Argentina, awareness of GEP-NET is significantly lower compared to that in the United States and Europe. No epidemiological survey or NET registry has yet been conducted, accounting for the total lack of accurate data on their incidence and prevalence. This lack of consensus on the diagnosis and management of GEP-NET in Argentina and the scarcity of data in our region (Latin America) (10), may account for their late diagnosis.

This study focuses on NET patients from Argentina. We decided to include patients with bronchial NET (BNET) (typical and atypical carcinoids only). Various treatment modalities, including surgery, nuclear radiation and systemic and targeted therapies, are topics of ongoing debate as practice patterns evolve. The management of toxicity, recurrence and progression is among the primary concerns of practitioners who make decisions on the clinical management of this relatively rare disease.
Data on this NET population may be of particular value in obtaining information on current and emerging treatment patterns in Argentina and providing insight into the optimal clinical practices.

The aim of this prospective study was to describe the clinical symptoms, diagnostic procedures, treatment and follow-up of NET in Argentina. This approach to routine clinical practice may enhance our understanding of and clinical experience with this uncommon tumor. To the best of our knowledge, this is the first study providing information on NET patients in Latin America.

\section{Patients and methods}

Study population. The population included in this study was obtained from a large database launched by a scientific work group in Argentina (the ARGENTUM group). Patients treated by oncologists, surgeons, or gastroenterologists and those seen at institutions affiliated with the ARGENTUM group were referred to the Bonorino Udaondo Hospital (Buenos Aires, Argentina) for inclusion in this observational study. The patients were categorized by tumor site and histological subtype, according to the latest WHO classification (6), as WDET, WDEC, PDEC, or mixed exocrine-endocrine carcinoma. All the cases were centrally evaluated (when necessary or required) by the pathologists in our group. The tissue samples were examined using hematoxylin and eosin and immunohistochemical staining techniques to confirm the diagnosis of GEP-NET.

Data collection. The medical records were reviewed to collect the following data: age, gender, functional syndrome, familial hereditary syndrome, diagnostic procedures, site and size of primary tumor, histopathological characteristics (based on the WHO classification), immunohistochemical staining, grade and tumor stage at diagnosis, metastatic pattern, therapeutic interventions and results (surgery, locoregional therapies, hormone therapy, chemotherapy, radiotherapy, radionuclide therapy and new targeted therapies). Other collected data included date of diagnosis and date of last visit or death.

The tumor stages were classified as locoregional (with invasion of surrounding organs or tissues or compromise of regional lymph nodes) or as distant metastases (the specific sites of distant metastases were also recorded). The most relevant clinical parameters were classified using descriptive statistics.

Statistical analysis. Data are described using averages and standard deviations for continuous variables with normal distribution or using medians and interquartile ranges (IQRs) in controversial cases. The distribution normality was assessed with the visual inspection of the box diagrams (box plots), histograms and quartile-quartile graphs and with the Shapiro-Wilk test. Categorical data were described using absolute numbers and percentages. The denominators used in the calculations are specified in each table $(\mathrm{n}=\mathrm{xxx})$.

To compare categorical variables, the $\chi^{2}$ test (with appropriate degrees of freedom according to the category of the variables compared) or the Fisher's exact test were used. To evaluate linear trend among categories, the Cochran-Armitage test was used. To compare continuous variables among groups, 
MOLECULAR AND CLINICAL ONCOLOGY 2: 673-684

675

A

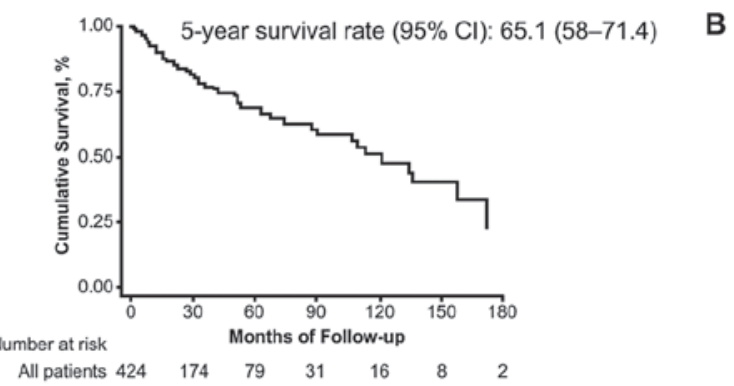

C

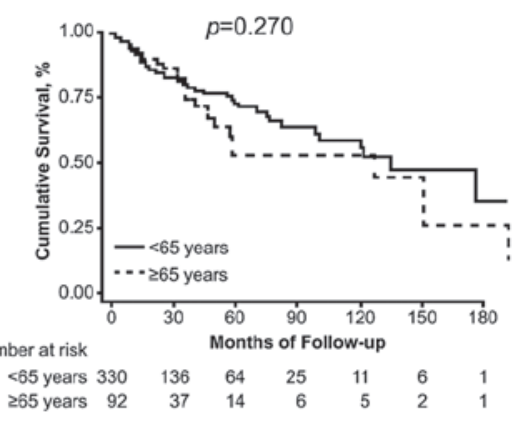

E

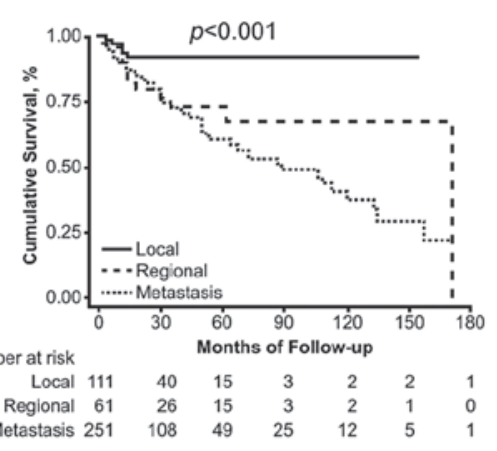

G
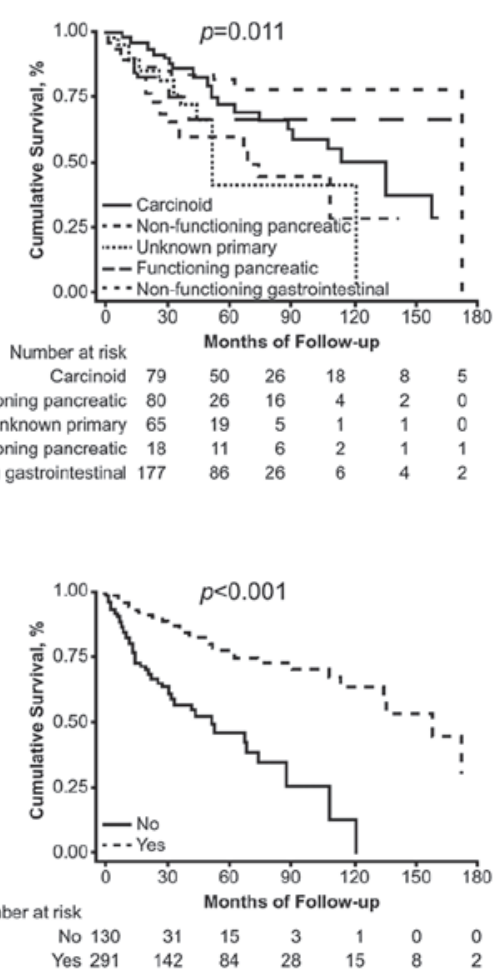

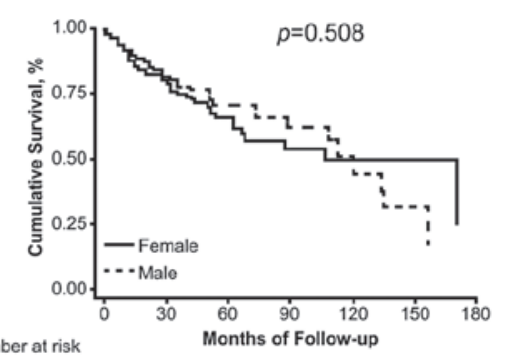

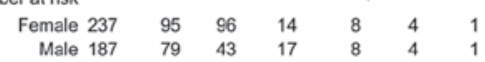

D

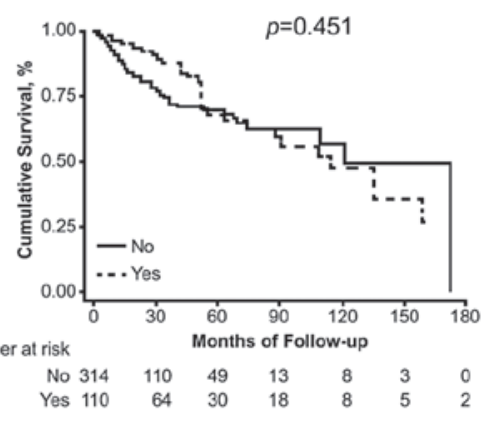

$\mathbf{F}$

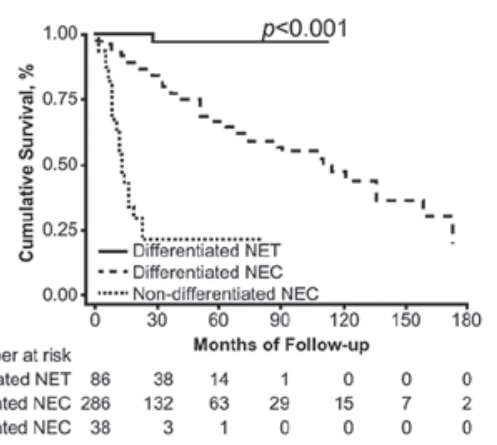

H
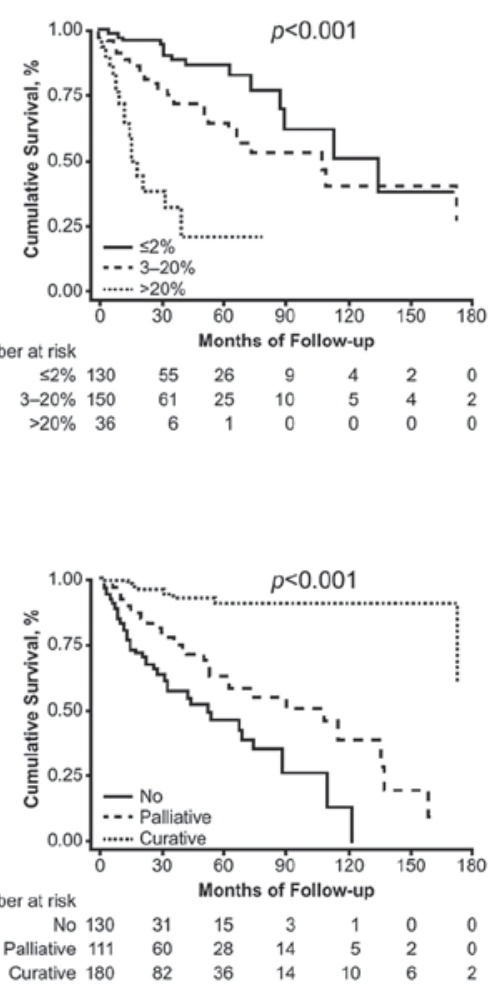

Figure 1. Overall survival (A) in all patients; (B) by gender; (C) by age; (D) by hormonal syndrome; (E) by stage; (F) by histological grade; (G) by tumor type; (H) by Ki-67; (I) by surgery; (J) by curative or palliative surgical intent. CI, confidence interval; NET, neuroendocrine tumor; NEC, neuroendocrine carcinoma. 
Table I. Characteristics of study population of GEP-NET.

\begin{tabular}{|c|c|c|c|c|}
\hline Variables & All patients $(n=461)$ GEP-NET & Female $(\mathrm{n}=255)$ & Male $(n=206)$ & P-value \\
\hline Age, years (SD), $(n=459)$ & $53.2(15.1)$ & $52.0(15.6)$ & $54.7(14.4)$ & $0.063^{\mathrm{a}}$ \\
\hline Male, $\mathrm{n}(\%),(\mathrm{n}=461)$ & $206(44.7)$ & - & - & - \\
\hline Carcinoid syndrome, $n(\%),(n=461)$ & $120(26.0)$ & $58(22.7)$ & $62(30.1)$ & $0.074^{\mathrm{b}}$ \\
\hline Incidental diagnosis, $n(\%),(n=434)$ & $49(10.6)$ & $28(11.0)$ & $21(10.2)$ & $0.785^{\mathrm{b}}$ \\
\hline \multicolumn{5}{|l|}{ Symptoms, n (\%), (n=434) } \\
\hline Diarrhea & $75(17.3)$ & $37(15.5)$ & $38(19.4)$ & $0.292^{\mathrm{b}}$ \\
\hline Abdominal pain & $139(32.0)$ & $80(33.6)$ & $59(30.1)$ & $0.435^{\mathrm{b}}$ \\
\hline Flushing & $19(4.4)$ & $10(4.2)$ & $9(4.6)$ & $0.843^{\mathrm{b}}$ \\
\hline Digestive hemorrhage & $19(4.4)$ & $8(3.4)$ & $11(5.6)$ & $0.254^{\mathrm{b}}$ \\
\hline Weight loss & $14(3.2)$ & $10(4.2)$ & $4(2.0)$ & $0.205^{\mathrm{b}}$ \\
\hline Intestinal obstruction & $29(6.7)$ & $15(6.3)$ & $14(7.1)$ & 0.727 \\
\hline \multicolumn{5}{|l|}{ Histological diagnosis, $n(\%),(n=457)$} \\
\hline Well-differentiated NET & $92(20.1)$ & $58(22.8)$ & $34(16.8)$ & $0.432^{\mathrm{b}}$ \\
\hline Well-differentiated NEC & $304(66.5)$ & $166(65.1)$ & $138(68.3)$ & \\
\hline Poorly differentiated NEC & $47(10.3)$ & $24(9.4)$ & $23(11.4)$ & \\
\hline Other & $14(3.1)$ & $7(2.7)$ & $7(3.5)$ & \\
\hline Hereditary syndrome, $\mathrm{n}(\%)^{\mathrm{c}},(\mathrm{n}=459)$ & $10(2.2)$ & $6(2.4)$ & $4(1.9)$ & $1.00^{\mathrm{d}}$ \\
\hline \multicolumn{5}{|l|}{ Staging at the time of diagnosis, $(n=461)$} \\
\hline Local & $118(25.6)$ & $75(29.4)$ & $43(20.8)$ & $0.062^{\mathrm{b}}$ \\
\hline Locally advanced & $68(14.8)$ & $39(15.3)$ & $29(14.1)$ & \\
\hline Metastatic & $273(59.2)$ & $141(55.3)$ & $132(64.1)$ & \\
\hline Not reported & $2(0.4)$ & $0(0.0)$ & $2(1.0)$ & \\
\hline Local tumors, n (\%) & $118(25.6)$ & $75(29.4)$ & $43(20.9)$ & $0.037^{\mathrm{b}}$ \\
\hline Size, median (IQR), (n=298) & $2.0(1.0-4.0)$ & $2.0(1.0-4.0)$ & $2.0(1.0-4.0)$ & $0.426^{\mathrm{e}}$ \\
\hline \multicolumn{5}{|l|}{ Size, $n(\%),(n=461)$} \\
\hline$\leq 2$ & $163(35.4)$ & $98(38.4)$ & $65(31.6)$ & $0.391^{\mathrm{b}, \mathrm{f}}$ \\
\hline$>2$ and $\leq 4$ & $68(14.7)$ & $36(14.1)$ & $32(15.5)$ & \\
\hline$>4$ & $67(14.5)$ & $38(14.9)$ & $29(14.1)$ & \\
\hline Not reported & $163(35.4)$ & $83(32.6)$ & $80(38.8)$ & \\
\hline \multicolumn{5}{|l|}{ Tumors, $n(\%),(n=461)$} \\
\hline Unicentric & $333(72.2)$ & $187(73.3)$ & $146(70.9)$ & $0.842^{\mathrm{b}}$ \\
\hline Multicentric & $45(9.8)$ & $24(9.4)$ & $21(10.2)$ & \\
\hline Not reported & $83(18.0)$ & $44(17.3)$ & $39(18.9)$ & \\
\hline \multicolumn{5}{|l|}{ Location, $\mathrm{n}(\%),\left(\mathrm{n}=461^{\mathrm{g}}\right)$} \\
\hline Esophagus & $13(2.8)$ & $7(2.7)$ & $6(2.9)$ & $0.914^{\mathrm{b}}$ \\
\hline Stomach & $32(6.9)$ & $17(6.7)$ & $15(7.3)$ & $0.796^{\mathrm{b}}$ \\
\hline Duodenum & $9(1.9)$ & $3(1.2)$ & $6(2.9)$ & $0.197^{\mathrm{d}}$ \\
\hline Small bowel & $124(26.9)$ & $62(24.3)$ & $62(30.1)$ & $0.164^{\mathrm{b}}$ \\
\hline Appendix & $35(7.6)$ & $24(9.4)$ & $11(5.4)$ & $0.101^{\mathrm{b}}$ \\
\hline Colon-rectum-anus & $57(12.4)$ & $38(14.9)$ & $19(9.2)$ & $0.066^{\mathrm{b}}$ \\
\hline Pancreas & $116(25.2)$ & $63(24.7)$ & $53(25.7)$ & $0.801^{\mathrm{b}}$ \\
\hline Unknown & $75(16.3)$ & $41(16.1)$ & $34(16.5)$ & $0.902^{\mathrm{b}}$ \\
\hline Ki-67, median (IQR), $(n=239)$ & $5.0(2.0-12.0)$ & $5.0(2.0-10.0)$ & $5.0(3.0-13.0)$ & 0.580 \\
\hline \multicolumn{5}{|l|}{ Ki-67 categories, $n(\%),(n=461)$} \\
\hline$\leq 2 \%$ & $135(29.3)$ & $84(32.9)$ & $51(24.8)$ & $0.212^{\mathrm{b}}$ \\
\hline $3-20 \%$ & $164(35.6)$ & $90(35.3)$ & $74(35.9)$ & \\
\hline$>20 \%$ & $41(8.9)$ & $20(7.8)$ & $21(10.2)$ & \\
\hline Not reported & $121(26.2)$ & $61(23.9)$ & $60(29.1)$ & \\
\hline \multicolumn{5}{|l|}{ Metastasis, $\mathrm{n}(\%),(\mathrm{n}=454)^{\mathrm{h}}$} \\
\hline Lymph nodes & $165(36.3)$ & $89(35.5)$ & $76(37.4)$ & $0.663^{\mathrm{b}}$ \\
\hline Liver & $254(55.9)$ & $131(52.2)$ & $123(60.6)$ & $0.073^{\mathrm{b}}$ \\
\hline
\end{tabular}


Table I. Continued.

\begin{tabular}{|c|c|c|c|c|}
\hline Variables & All patients $(n=461)$ GEP-NET & Female $(n=255)$ & Male $(n=206)$ & P-value \\
\hline Peritoneum & $49(10.8)$ & $25(10.0)$ & $24(11.8)$ & $0.525^{\mathrm{b}}$ \\
\hline Lung & $10(2.2)$ & $5(2.0)$ & $5(2.5)$ & $0.758^{\mathrm{e}}$ \\
\hline Bone & $5(1.1)$ & $2(0.8)$ & $3(1.5)$ & $0.660^{\mathrm{d}}$ \\
\hline Other & $12(2.6)$ & $8(3.2)$ & $4(2.0)$ & $0.422^{\mathrm{b}}$ \\
\hline $\begin{array}{l}\text { Delay from onset of symptoms to diagnosis, } \\
\text { in months, median (IQR), }(n=282)\end{array}$ & $9.0(3.0-24.0)$ & $10.0(3.0-27.0)$ & $8.0(3.25-20.0)$ & 0.467 \\
\hline
\end{tabular}

${ }^{a}$ Student's t-test. ${ }^{b} \chi^{2}$ test. ${ }^{c}$ Six patients with multiple endocrine neoplasia 1 (MEN-1), 2 with neurofibromatosis type 1,1 with tuberous sclerosis complex and 1 with von Hippel-Lindau disease. ${ }^{d}$ Fisher's exact test. ${ }^{e}$ Mann-Whitney U test. ${ }^{\mathrm{f}} \mathrm{P}=0.592$, excluding patients not reported. ${ }^{\mathrm{g}}$ There were 6 patients characterized as 'primary unknown=no' but presenting with a non-specific location, who were analyzed as having an unknown

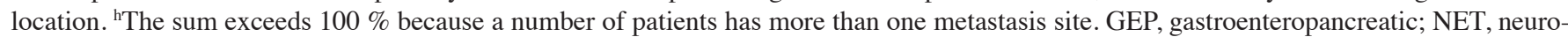
endocrine tumor; SD, standard deviation; NEC, neuroendocrine carcinoma; IQR, interquartile range.

Table II. Comparison between WHO $2000(6,11)$ and WHO 2010 (12) classifications of GEP-NET ${ }^{\mathrm{a}}$.

\begin{tabular}{|c|c|c|c|c|c|}
\hline \multirow[b]{2}{*}{ GEP-NET } & \multirow[b]{2}{*}{ WHO 2000 classification, n $(\%)^{\mathrm{b}}$} & \multicolumn{4}{|c|}{ WHO 2010 classification, n (\%) } \\
\hline & & No data ${ }^{c}$ & NET G1 & NET G2 & NEC G3 \\
\hline WDET & $92(20.1)$ & $28(30.4)$ & $51(55.5)$ & $13(14.1)$ & $0(0.0)$ \\
\hline WDEC & $304(66.5)$ & $72(23.7)$ & $82(27.0)$ & $144(47.4)$ & $6(1.9)$ \\
\hline PDEC & $47(10.3)$ & $11(23.4)$ & $0(0.0)$ & $4(8.5)$ & $32(68.1)$ \\
\hline Others & $14(3.1)$ & $6(42.9)$ & $2(14.3)$ & $3(21.4)$ & $3(21.4)$ \\
\hline Total & $457(100.0)$ & $117(25.6)$ & $135(29.5)$ & $164(35.9)$ & $41(9.0)$ \\
\hline
\end{tabular}

${ }^{a}$ The table is based on 457 patients for whom data were available. ${ }^{b}$ The percentages in the second column are percentages of the total number of classified patients. 'In the 'No data' columns, G1, G2 and G3 are percentages of each line of NET and NEC, differentiated neuroendocrine tumor (NET) G1, G2 and neuroendocrine carcinoma (NEC) G3. WHO, World Health Organization; GEP, gastroenteropancreatic; NET, neuroendocrine tumor; G1, grade 1; G2, grade 2; G3, grade 3; WDET, well-differentiated NET; WDEC, well-differentiated NEC; PDEC, poorly differentiated NEC.

the Student's t-test was used for variables with a normal distribution. Otherwise, the Mann-Whitney U test was used to compare two groups and the Kruskal-Wallis test was used to analyze more than two groups. Cumulative survival and survival graphs were calculated with the Kaplan-Meier method and survival functions in different groups were compared using the log-rank test. All the tests were two-tailed and $\mathrm{P}<0.05$ was considered an indicator of statistically significant differences. No adjustments were made for multiple comparisons.

\section{Results}

Patient population. Of the 532 assessable patients with a diagnosis of NET, $461(86.6 \%)$ had GEP-NET and $71(13.4 \%)$ had BNET. In the primary analysis, the GEP-NET data were used. The median age at diagnosis was 53.2 years and $26.0 \%$ of the GEP-NET patients presented with hormone hypersecretion symptoms, with no significant differences according to gender. Hereditary syndromes associated with NET were diagnosed in 10 patients $(2.2 \%)$, the majority of whom were women (Table I). Of the 71 patients with NET of bronchopulmonary origin, 43 were women $(61.0 \%)$ and 28 were men
(39.0\%); their median age was 38 years (range, 13-67 years) (data not shown).

According to the WHO 2000 classification $(6,11)$, 92 patients $(20.1 \%)$ with NET in various locations were classified as WDET, 304 (66.5\%) as WDEC and 47 (10.3\%) as PDEC. According to the current (2010) WHO classification (12), 135 $(29.5 \%)$ of the 457 patients were reclassified as NET grade 1 (G1), $164(35.9 \%)$ as NET G2 and $41(9.0 \%)$ as neuroendocrine carcinoma G3 (Table II).

Diagnostic procedures. Incidental diagnosis occurred in $10.6 \%$ of the patients (Table I). The diagnoses were made primarily by performing incisional and excisional biopsies in 30.1 and $59.6 \%$ of the patients, respectively. In addition, $18.1 \%$ of the patients required more than one diagnostic procedure (repeat biopsy). Biochemical tests, such as measurement of serum chromogranin A and urinary 5-hydroxyindoleacetic acid levels, were performed in 72.8 and $41.7 \%$ of the patients, respectively. Scintigraphy with radiolabeled octreotide (octreoscan) was performed in $46.5 \%$ of the patients, with positive results in $79.0 \%$ of the cases. Immunohistochemical staining for chromogranin A and synaptophysin was performed in 85.6 and 
Table III. Diagnostic procedures.

Procedures Patients

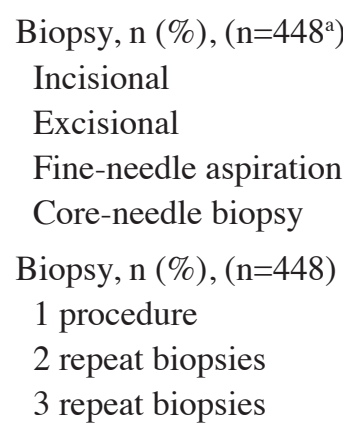

Immunohistochemistry, $\mathrm{n}(\%),(\mathrm{n}=459)$

Synaptophysin
Performed
Positive
Negative
Not performed
Chromogranin A
Performed
Positive
Negative
Not performed
TTF-1
Performed
Positive
Negative
Not performed
Biopsy material, $\mathrm{n}(\%),(\mathrm{n}=458)$
Primary tumor
Primary tumor + metastasis
Metastasis
Biopsy location, $\mathrm{n}(\%),(\mathrm{n}=457)$
Intestines
Liver
Lymph nodes
Lung
Other

Diagnostic studies, n (\%)

$\begin{array}{lc}\text { Chromogranin A in serum }(\mathrm{n}=459) & \\ \text { Yes } & 334(72.8) \\ \text { No } & 104(22.6) \\ \text { Not performed } & 21(4.6) \\ \text { 5-HIAA }(\mathrm{n}=460) & \\ \text { Performed } & 192(41.7) \\ \text { Positive } & 71(15.4)\left(37.0^{\mathrm{b}}\right) \\ \text { Negative } & 121(26.3)\left(63.0^{\mathrm{b}}\right) \\ \text { Not performed } & 268(58.3) \\ \text { Peptides }(\mathrm{n}=460) & \\ \text { Performed } & 107(23.3) \\ \text { Positive } & 43(9.4)\left(40.2^{\mathrm{b}}\right) \\ \text { Negative } & 64(13.9)(50.8 \mathrm{~b}) \\ \text { Not performed } & 353(76.7)\end{array}$

$338(73.6)\left(86.0^{\mathrm{b}}\right)$

$55(12.0)\left(14.0^{\mathrm{b}}\right)$

$66(14.4)$

$33(7.2)$

$3(0.7)\left(9.1^{\mathrm{b}}\right)$

$30(6.5)\left(90.9^{b}\right)$

426 (92.8)

$227(49.6)$

95 (20.7)

136 (29.7)

$183(40.1)$

$124(27.1)$

18 (3.9)

132 (28.9)
Table III. Continued.

\begin{tabular}{lc}
\hline Procedures & Patients \\
\hline Octreoscan $(\mathrm{n}=460)$ & \\
Performed & $214(46.5)$ \\
Positive & $169(36.7)\left(79.0^{\mathrm{b}}\right)$ \\
Negative & $45(9.8)\left(21.0^{\mathrm{b}}\right)$ \\
Not performed & $246(53.5)$ \\
Ki67 (n=461) & \\
Reported & $340(73.8)$ \\
Not reported & $121(26.2)$ \\
Echocardiogram (n=460) & \\
Performed & $140(30.4)$ \\
Carcinoid-positive & $31(6.7)\left(22.1^{\mathrm{b}}\right)$ \\
Carcinoid-negative & $109(23.7)\left(77.9^{\mathrm{b}}\right)$ \\
Not performed & $320(69.6)$ \\
\hline
\end{tabular}

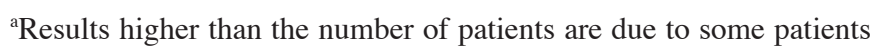
undergoing $>1$ procedure. ${ }^{b}$ Percentages of patients in whom the studies were conducted. TTF-1, thyroid transcription factor; 5-HIAA, 5-hydroxyindoleacetic acid.

$79.9 \%$ of the tumors. The Ki-67 index was calculated in $73.7 \%$ of the tumors. Echocardiography was performed in $30.4 \%$ of the reported cases and the results were positive in $22.1 \%$ of the cases. The diagnostic procedures are summarized in Table III.

Tumor characteristics. Of the patients with GEP-NET, 118 $(25.6 \%)$ had local tumors at diagnosis, 68 (14.8\%) had locally advanced disease and $273(59.2 \%)$ had distant metastases. Over $35.4 \%$ of the patients with GEP-NET had tumors sized $<2 \mathrm{~cm}$, whereas 68 of the tumors $(14.8 \%)$ were sized $2-4 \mathrm{~cm}$ and $14.5 \%$ were $>4 \mathrm{~cm}$. We were unable to obtain information regarding tumor size in 163 patients (35.4\%). In $9.8 \%$ of the patients the tumors were multicentric. The Ki-67 index was $\leq 2 \%$ in $29.3 \%, 3-20 \%$ in $35.6 \%$ and $>20 \%$ in $8.9 \%$ of the assessed tumors (Table I). In BNET, 63 patients (89.0\%) had typical and 8 had atypical carcinoids. Nine typical $(15.0 \%)$ and 5 atypical carcinoids $(63.0 \%)$ presented with N2 lymph node metastasis $(\mathrm{P}=0.002)$, with median tumor sizes of $2.8 \mathrm{~cm}$ (range, $5-7 \mathrm{~cm}$ ) and $3.25 \mathrm{~cm}$ (range, 2-5 cm), respectively (data not shown).

We analyzed metastatic sites in patients with GEP-NET (Table IV). Hepatic compromise was observed in $56.0 \%$ of the patients, followed by lymph node compromise in $36.3 \%$, peritoneum in $11.0 \%$, lung in $2.2 \%$ and bone in $1.1 \%$ of the cases. A 9-month delay from the onset of symptoms to the time of diagnosis was observed; this information, not often described in current studies, is important, although interpretation bias may limit its value.

Staging at diagnosis was significantly different, depending on the primary tumor location, tumor type and grade (Table V). The most common tumor types were gastrointestinal carcinoids $(59.0 \%)$, followed by pancreatic endocrine tumors $(25.0 \%)$ and metastatic NEC of unknown primary location (16.0\%). Functioning pancreatic endocrine tumors were identified in 
Table IV. Locations of metastases and primary tumors in GEP-NET.

\begin{tabular}{|c|c|c|c|c|c|c|c|c|c|}
\hline \multirow[b]{2}{*}{$\begin{array}{l}\text { Locations of } \\
\text { metastases, n (\%) }\end{array}$} & \multicolumn{8}{|c|}{ Locations of primary tumor ${ }^{\mathrm{a}}, \mathrm{n}(\%)$} & \multirow[b]{2}{*}{ P-value } \\
\hline & $\begin{array}{l}\text { Esophagus } \\
\qquad(\mathrm{n}=13)\end{array}$ & $\begin{array}{l}\text { Stomach } \\
(\mathrm{n}=32)\end{array}$ & $\begin{array}{l}\text { Duodenum } \\
\qquad(\mathrm{n}=9)\end{array}$ & $\begin{array}{l}\text { Small bowel } \\
\qquad(\mathrm{n}=124)\end{array}$ & $\begin{array}{c}\text { Pancreas } \\
(n=116)\end{array}$ & $\begin{array}{l}\text { Appendix } \\
(\mathrm{n}=35)\end{array}$ & $\begin{array}{l}\text { Colon } \\
(n=16)\end{array}$ & $\begin{array}{l}\text { Rectum } \\
(\mathrm{n}=41)\end{array}$ & \\
\hline Lymph nodes & $4(30.8)$ & $6(18.8)$ & $2(22.2)$ & $74(59.7)$ & $44(37.9)$ & $3(8.6)$ & $7(43.8)$ & $5(12.2)$ & $<0.001$ \\
\hline Liver & $3(23.1)$ & $5(15.6)$ & $3(33.3)$ & $87(70.2)$ & $74(63.8)$ & $0(0.0)$ & $11(68.8)$ & $5(12.2)$ & $<0.001$ \\
\hline Peritoneum & $0(0.0)$ & $0(0.0)$ & $2(22.2)$ & $33(26.6)$ & $5(4.3)$ & $0(0.0)$ & $1(6.2)$ & $0(0.0)$ & $<0.001$ \\
\hline Lung & $1(7.7)$ & $0(0.0)$ & $0(0.0)$ & $0(0.0)$ & $4(3.4)$ & $0(0.0)$ & $0(0.0)$ & $0(0.0)$ & 0.109 \\
\hline Bone & $0(0.0)$ & $0(0.0)$ & $0(0.0)$ & $1(0.8)$ & $2(1.7)$ & $0(0.0)$ & $0(0.0)$ & $0(0.0)$ & 0.928 \\
\hline Ovary & $0(0.0)$ & $0(0.0)$ & $0(0.0)$ & $2(1.6)^{b}$ & $0(0.0)$ & $0(0.0)$ & $0(0.0)$ & $0(0.0)$ & 0.751 \\
\hline Brain & $0(0.0)$ & $0(0.0)$ & $0(0.0)$ & $0(0.0)$ & $1(0.9)$ & $0(0.0)$ & $0(0.0)$ & $0(0.0)$ & 0.939 \\
\hline Liver + other & $1(7.7)$ & $2(6.2)$ & $0(0.0)$ & $66(53.2)$ & $32(27.6)$ & $0(0.0)$ & $5(31.2)$ & $2(4.9)$ & $<0.001$ \\
\hline
\end{tabular}

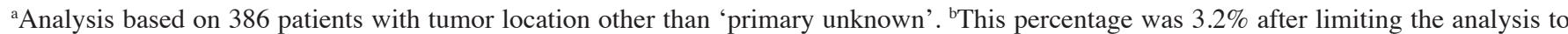
female patients (62 small bowel tumors in women). GEP, gastroenteropancreatic; NET, neuroendocrine tumor.

Table V. Staging at time of diagnosis according to location of primary tumor and tumor type in GEP-NET.

\begin{tabular}{|c|c|c|c|c|c|}
\hline Characteristics $^{\mathrm{a}}$ & $\begin{array}{c}\text { All } \\
(n=459)\end{array}$ & $\begin{array}{l}\text { Local tumors } \\
\qquad(\mathrm{n}=118)\end{array}$ & $\begin{array}{l}\text { Regional invasion } \\
\qquad(\mathrm{n}=68)\end{array}$ & $\begin{array}{l}\text { Metastatic } \\
(n=273)\end{array}$ & P-value \\
\hline Tumor type, $\mathrm{n}(\%),(\mathrm{n}=459)$ & & & & & $<0.001$ \\
\hline Intestinal & $270(100.0)$ & $102(37.8)$ & $39(14.4)$ & $129(47.8)$ & \\
\hline Non-functioning pancreatic & $106(100.0)$ & $15(14.2)$ & $26(24.5)$ & $65(61.3)$ & \\
\hline Functioning pancreatic & $10(100.0)$ & $1(10.0)$ & $0(0.0)$ & $9(90.0)$ & \\
\hline Primary unknown & $73(100.0)$ & - & $3(4.1)^{\mathrm{b}}$ & $70(95.9)$ & \\
\hline \multicolumn{6}{|l|}{ Tumor site $(n=459)$} \\
\hline Gastrointestinal tract & $270(100.0)$ & $102(37.8)$ & $39(14.4)$ & $129(47.8)$ & $<0.001$ \\
\hline Esophagus & $13(100.0)\left(4.8^{\mathrm{c}}\right)$ & $5(38.4)\left(4.9^{c}\right)$ & $4(30.8)\left(10.3^{c}\right)$ & $4(30.8)\left(3.1^{\mathrm{c}}\right)$ & 0.082 \\
\hline Stomach & $32(100.0)\left(11.9^{c}\right)$ & $22(68.8)\left(21.6^{\mathrm{c}}\right)$ & $4(12.5)\left(10.3^{c}\right)$ & $6(18.7))\left(4.7^{c}\right)$ & $<0.001$ \\
\hline Duodenum & $9(100.0)\left(3.3^{c}\right)$ & $3(33.3)\left(2.9^{c}\right)$ & $1(11.1)\left(2.5^{c}\right)$ & $5(55.6)\left(3.9^{c}\right)$ & 0.854 \\
\hline Jejunum-ileum & $124(100.0)\left(45.9^{c}\right)$ & $7(5.7)\left(6.9^{c}\right)$ & $23(18.5)\left(58.9^{c}\right)$ & $94(75.8)\left(72.9^{c}\right)$ & $<0.001$ \\
\hline Appendix & $35(100.0)\left(13.0^{c}\right)$ & $30(85.7)\left(29.4^{c}\right)$ & $3(8.6)\left(7.7^{c}\right)$ & $2(5.7)\left(1.5^{\mathrm{c}}\right)$ & $<0.001$ \\
\hline Colon-rectum & $57(100.0)\left(21.1^{\mathrm{c}}\right)$ & $35(61.4)\left(34.3^{\mathrm{c}}\right)$ & $4(7.0)\left(10.3^{c}\right)$ & $18(31.6)\left(13.9^{c}\right)$ & $<0.001$ \\
\hline Pancreas & $116(100.0)$ & $16(13.8)$ & $26(22.4)$ & $74(63.8)$ & 0.001 \\
\hline Unknown & $73(100.0)$ & - & $3(4.1)$ & $70(95.9)$ & $<0.001$ \\
\hline \multicolumn{6}{|l|}{ Histopathology, $n(\%),(n=457)$} \\
\hline$\leq 2 \%$ & $135(100.0)$ & $53(39.3)$ & $17(12.6)$ & $65(48.1)$ & $<0.001$ \\
\hline $3-20 \%$ & $164(100.0)$ & $26(15.9)$ & $23(14.0)$ & $115(70.1)$ & \\
\hline$>20 \%$ & $41(100.0)$ & $5(12.2)$ & $9(21.9)$ & $27(65.9)$ & \\
\hline Not performed & $117(100.0)$ & $33(28.2)$ & $19(16.2)$ & $65(55.6)$ & \\
\hline Ki-67, median (IQR), $(n=239)$ & $5.0(2.0-12.0)$ & $3.0(2.0-9.5)$ & $6.0(3.0-21.0)$ & $5.0(3.0-12.0)$ & $0.02^{\mathrm{d}}$ \\
\hline \multicolumn{6}{|l|}{$\begin{array}{l}\text { Tumor differentiation degree, } \\
\mathrm{n}(\%),(\mathrm{n}=456)\end{array}$} \\
\hline Well-differentiated NET & $92(100.0)$ & $79(85.9)$ & $3(3.2)$ & $10(10.9)$ & $<0.001$ \\
\hline Differentiated NEC & $303(100.0)$ & $22(7.3)$ & $52(17.1)$ & $229(75.6)$ & \\
\hline Poorly differentiated NEC & $47(100.0)$ & $8(17.0)$ & $12(25.5)$ & $27(57.5)$ & \\
\hline Other & $14(100.0)$ & $8(57.24)$ & $1(7.1)$ & $5(35.7)$ & \\
\hline $\begin{array}{l}\text { Delay from symptom onset to } \\
\text { time of diagnosis, in months, } \\
\text { median (IQR), }(n=282)\end{array}$ & $9.0(3.0-24.0)$ & $8.0(4.0-18.0)$ & $8.5(2.3-25.3)$ & $10.0(3.0-27.0)$ & 0.876 \\
\hline
\end{tabular}


Table VI. Therapeutic interventions for patients with GEP-NET.

\begin{tabular}{|c|c|c|c|c|c|}
\hline Interventions & All (n=459) & $\begin{array}{l}\text { Local tumor } \\
\quad(\mathrm{n}=118)\end{array}$ & $\begin{array}{l}\text { Regional invasion } \\
(\mathrm{n}=68)\end{array}$ & $\begin{array}{l}\text { Metastatic } \\
(\mathrm{n}=273)\end{array}$ & P-value \\
\hline \multicolumn{6}{|l|}{ Surgery, n (\%), (n=309) } \\
\hline & & $<0.001$ & & & \\
\hline Curative & $189(100.0)$ & $103(54.5)$ & $45(23.8)$ & $41(21.7)$ & \\
\hline Palliative & $120(100.0)$ & $1(0.8)$ & $9(7.5)$ & $110(91.7)$ & \\
\hline Regional procedure, $n(\%),(n=459)$ & $40(100.0)$ & $0(0.0)$ & $1(2.5)$ & $39(97.5)$ & $<0.001^{\mathrm{a}}$ \\
\hline Chemoembolization & $24(100.0)$ & $0(0.0)$ & $1(4.2)$ & $23(95.8)$ & $0.877^{\mathrm{b}}$ \\
\hline Radiofrequency & $7(100.0)$ & $0(0.0)$ & $0(0.0)$ & $7(100.0)$ & \\
\hline Embolization & $4(100.0)$ & $0(0.0)$ & $0(0.0)$ & $4(100.0)$ & \\
\hline Other & $5(100.0)$ & $0(0.0)$ & $0(0.0)$ & $5(100.0)$ & \\
\hline $\begin{array}{l}\text { Radiopharmaceuticals, n (\%) } \\
(\mathrm{n}=459)\end{array}$ & $8(100.0)$ & $0(0.0)$ & $0(0.0)$ & $8(100.0)$ & 0.062 \\
\hline Radiotherapy, $\mathrm{n}(\%),(\mathrm{n}=421)$ & $30(100.0)$ & $5(16.7)$ & $9(30.0)$ & $16(53.3)$ & 0.045 \\
\hline \multicolumn{6}{|l|}{ Medical treatment, $\mathrm{n}(\%),(\mathrm{n}=458)$} \\
\hline Analogues and/or interferon & $461(100.0)$ & $144(31.2)$ & $62(13.5)$ & $255(55.3)$ & $0.810^{\mathrm{a}}$ \\
\hline Analogue-only receivers & $140(100.0)\left(30.3^{c}\right)$ & $0(0.0)$ & $12(8.6)\left(19.4^{\mathfrak{c}}\right)$ & $128(91.4)\left(50.1^{\mathrm{c}}\right)$ & $<0.001^{b}$ \\
\hline Interferon-only receivers & $41(100.0)\left(8.9^{c}\right)$ & $0(0.0)$ & $0(0.0)$ & $41(100.0)\left(16.1^{c}\right)$ & \\
\hline Both & $22(100.0)\left(4.8^{c}\right)$ & $0(0.0)$ & $3(13.6)\left(4.8^{c}\right)$ & $19(86.4)\left(7.4^{c}\right)$ & \\
\hline Chemotherapy, n (\%), (n=456) & $133(100.0)$ & $10(7.5)$ & $18(13.5)$ & $105(79.0)$ & $<0.001^{\mathrm{d}}$ \\
\hline \multicolumn{6}{|l|}{ Chemotherapy cycles, $n(\%),\left(n=123^{\mathrm{e}}\right)$} \\
\hline 1 cycle & $94(100.0)$ & $10(10.7)$ & $13(13.8)$ & $71(75.5)$ & 0.246 \\
\hline 2 cycles & $19(100.0)$ & $0(0.0)$ & $2(10.5)$ & $17(89.5)$ & \\
\hline 3 cycles & $10(100.0)$ & $0(0.0)$ & $3(30.0)$ & $7(70.0)$ & \\
\hline Sunitinib, $\mathrm{n}(\%),(\mathrm{n}=422)$ & $21(100.0)$ & $0(0.0)$ & $2(9.5)$ & $19(90.5)$ & $0.086^{\mathrm{f}}$ \\
\hline Everolimus, $\mathrm{n}(\%),(\mathrm{n}=423)$ & $15(100.0)$ & $0(0.0)$ & $1(6.6)$ & $14(93.4)$ & $0.048^{\mathrm{f}}$ \\
\hline New drugs, $n(\%),(n=419)$ & $36(100.0)$ & $0(0.0)$ & $3(8.3)$ & $33(91.7)$ & $0.004^{\mathrm{a}}$ \\
\hline
\end{tabular}

${ }^{\mathrm{a}} \chi^{2}$ test on distribution of use or non-use of regional therapies. ${ }^{\mathrm{b}} \chi^{2}$ test on distribution of therapies used among patients who were effectively treated. ${ }^{~ P e r c e n t a g e ~ o f ~ t r e a t e d ~ p a t i e n t s . ~}{ }^{\mathrm{d} P}$-value for $\chi^{2}$ test and for Cochran-Armitage test (linear trend test). ${ }^{\mathrm{e}} \mathrm{Based}$ on patients with complete information on the 3 chemotherapy cycles (no patients received 4 cycles); the absence of data explains why information on the 3 cycles was only available for 123 patients, although 133 patients received at least 1 chemotherapy cycle. ${ }^{\mathrm{f}} \mathrm{P}$-value for $\chi^{2}$ test. $\mathrm{P}=0.062$ for Cochran-Armitage test. GEP, gastroenteropancreatic; NET, neuroendocrine tumors.

$8.62 \%$ of all patients with pancreatic NET (pNET) and in $2.1 \%$ of the total population. The gastrointestinal tract was the primary tumor site in 270 patients $(58.6 \%)$ and the pancreas in 116 patients $(25.2 \%)$; in 73 patients $(15.9 \%)$, the primary tumor site was unknown. Among gastrointestinal tumors, the small bowel (45.9\%), colon-rectum (21.1\%), appendix (13.0\%) and stomach $(11.9 \%)$ were the most frequent sites of origin (Table V).

The small bowel was the primary tumor site in the majority $(75.8 \%)$ of patients with a higher incidence of hepatic involvement. In NET of gastrointestinal origin, the esophagus (30.8\%) and the colon-rectum (13.9\%) were most frequently involved. The majority $(63.8 \%)$ of patients with pNET had metastases at diagnosis. Functioning pancreatic tumors $(90.0 \%)$ were those most commonly associated with widespread disease. A different pattern was observed in patients with primary tumors of the appendix and the stomach. Only 1.5 and $4.7 \%$ of those patients, respectively, had tumors that had already metastasized at the time of diagnosis. A significant proportion $(57.4 \%)$ of patients with PDEC had metastases, as did $75.6 \%$ of WDEC and $10.9 \%$ of WDET patients.

Therapeutic interventions. Overall, $67.3 \%$ of the GEP-NET patients underwent surgery, $41.2 \%$ with curative intent and $26.1 \%$ for palliative purposes. Curative intent surgery was more frequent in patients with local disease (54.5\%) and locally advanced disease $(23.8 \%)$. Palliative surgery was performed in $91.7 \%$ of patients with metastatic disease. Locoregional therapies, such as embolization, chemoembolization, radiofrequency and other ablative techniques were uncommon (8.7\%); chemoembolization was the mostly frequently used locoregional therapy (60.0\% of regional therapies) (Table VI).

A total of 203 patients received some type of systemic treatment, including chemotherapy received by 133 patients, during the course of their disease: somatostatin analogues 
Table VII. Survival and prognostic factors in GEP-NET.

\begin{tabular}{|c|c|c|c|c|c|}
\hline Patients & No. & Median (months) & 5-year survival rate $(\%)$ & $95 \%$ confidence interval & P-value \\
\hline All & 424 & 121 & 65.1 & $58.0-71.4$ & - \\
\hline Gender & 424 & & & & \\
\hline Female & 237 & 108 & 62.1 & $51.7-70.9$ & 0.508 \\
\hline Male & 187 & 121 & 68.3 & $58.0-76.7$ & \\
\hline Age (years) & 422 & & & & \\
\hline$\geq 65$ & 92 & 114 & 50.7 & $34.2-65.1$ & 0.270 \\
\hline$<65$ & 330 & 121 & 69.1 & $61.4-75.7$ & \\
\hline Carcinoid syndrome & 424 & & & & \\
\hline Yes & 110 & 114 & 64.5 & $50.4-75.6$ & 0.451 \\
\hline No & 314 & 121 & 66.5 & $58.4-73.4$ & \\
\hline Stage at diagnosis & 423 & & & & \\
\hline Local & 111 & $\mathrm{NS}^{\mathrm{a}}$ & 91.8 & $82.5-96.3$ & $<0.001$ \\
\hline Regionally advanced & 61 & 172 & 68.3 & $50.2-81.0$ & \\
\hline Metastatic & 251 & 90 & 55.9 & $46.7-64.2$ & \\
\hline Location & 358 & & & & \\
\hline Gastrointestinal tract & 253 & 136 & 74.0 & $65.1-80.9$ & 0.011 \\
\hline Pancreas & 105 & 74 & 56.3 & $42.6-67.9$ & \\
\hline Tumor type & 421 & & & & \\
\hline Carcinoid & 79 & 135 & 68.0 & $52.4-79.4$ & 0.011 \\
\hline Non-functioning pancreatic & 80 & 68 & 55.1 & $38.7-68.9$ & \\
\hline Primary unknown & 66 & 52 & 38.1 & $16.0-60.3$ & \\
\hline Functioning pancreatic non-carcinoid & 19 & $\mathrm{NS}^{\mathrm{a}}$ & 63.3 & $34.8-82.1$ & \\
\hline Non-functioning gastrointestinal & 177 & 172 & 78.8 & $68.7-86.0$ & \\
\hline Ki-67 category & 316 & & & & \\
\hline$\leq 2 \%$ & 130 & 135 & 85.0 & $72.2-92.2^{b}$ & $<0.001$ \\
\hline $3-20 \%$ & 150 & 108 & 61.7 & $49.8-71.6^{\mathrm{b}}$ & \\
\hline$>20 \%$ & 36 & 16 & 15.7 & $3.2-36.9^{\mathrm{b}}$ & \\
\hline Binary Ki-67 & 220 & & & & \\
\hline$\geq 5$ & 125 & 63 & 47.1 & $34.7-58.5^{\mathrm{b}}$ & $<0.001$ \\
\hline$<5$ & 95 & 172 & 85.7 & $71.4-93.2^{\mathrm{b}}$ & \\
\hline Tumor grade & 410 & & & & \\
\hline Differentiated NET & 86 & $\mathrm{NS}^{\mathrm{a}}$ & 97.4 & $82.8-99.6$ & $<0.001$ \\
\hline Differentiated NEC & 286 & 114 & 63.6 & $54.8-71.0$ & \\
\hline Poorly differentiated NEC & 38 & 13 & 18.4 & $5.7-36.7$ & \\
\hline
\end{tabular}

${ }^{\text {an }}$ Not estimable due to the small number of events. 'Interquartile range. GEP, gastroenteropancreatic; NET, neuroendocrine tumors; NEC, neuroendocrine carcinoma.

$(30.3 \%)$, interferon $(8.9 \%)$ and combined treatment with somatostatin analogues plus interferon (4.8\%). A total of 94 patients $(29.0 \%)$ underwent at least one chemotherapy cycle and $36(7.8 \%)$ received a new drug (everolimus or sunitinib). These percentages were higher among patients with advanced disease (somatostatin analogues, 91.4\%; interferon, $100.0 \%$; combined treatment, $86.3 \%$ ). A total of $75.5 \%$ of patients who underwent chemotherapy had advanced disease, as did $91.6 \%$ of patients treated with new drugs (Table VI).

Survival and prognostic factors. The association between the overall survival and prognostic factors is summarised in Fig. 1.
The overall survival of the GEP-NET patients in this study was 65.1 months (range, 58-71.4 months). A total of 46 patients $(10.0 \%)$ were given different histological diagnoses following examination by the pathologists in our group: hepatocellular carcinoma (13.04\%), poorly differentiated carcinoma $(8.7 \%)$, lymphoproliferative disease $(8.7 \%)$, primary unknown adenocarcinoma $(6.5 \%)$, colon adenocarcinoma (6.5\%) and not otherwise specified (61.0\%) (data not shown). Based on epidemiological data from SEER (1950-1999) (5), 22.4\% of the cases of non-carcinoid second primary tumors have been described in patients with diagnoses of NET; a high percentage of associated tumors occurred in small intestinal carcinoids (29.0\%). 
Table VIII. Cox multivariate analysis for overall survival in GEP-NET (model 1 ${ }^{\mathrm{a}}$, $\mathrm{n}=376$ ).

\begin{tabular}{lccr}
\hline Variables & Odds ratio & $95 \%$ confidence interval & P-value \\
\hline Age & 0.99 & $0.97-1.01$ & 0.583 \\
Male gender & 0.90 & $0.53-1.53$ & 0.706 \\
Tumor stage & (Reference) & - & - \\
Local & 2.44 & $0.73-8.23$ & 0.150 \\
Regional & 3.12 & $1.02-9.56$ & 0.047 \\
Metastatic & & & - \\
Tumor type & (Reference) & - & 0.483 \\
Carcinoid & 0.75 & $0.33-1.69$ & 0.018 \\
Non-functioning pancreatic & 0.33 & $0.13-0.83$ & 0.338 \\
Primary unknown & 0.58 & $0.19-1.79$ & 0.027 \\
Functioning pancreatic non-carcinoid & 0.39 & $0.17-0.90$ & - \\
Non-functioning gastrointestinal & & & 0.023 \\
Tumor grade & (Reference) & - & $<0.001$ \\
Differentiated NET & 11.40 & $1.40-92.68$ & 0.008 \\
Differentiated NEC & 67.41 & $7.95-571.67$ & $0.23-0.81$ \\
Non-differentiated & 0.43 & & \\
Surgery & & & \\
\hline
\end{tabular}

${ }^{\text {a}}$ Variables included in the model were age, gender, stage at diagnosis, tumor subtype, tumor grade and evidence of surgery. All are included in the table. GEP, gastroenteropancreatic; NET, neuroendocrine tumors; NEC, neuroendocrine carcinoma.

The incidence of this type of tumors varies, depending on the series assessed.

Synchronous or metachronous second tumors developed in 50 patients $(11.0 \%)$ : colorectal $(24.0 \%)$, prostate $(14.0 \%)$, breast $(6.0 \%)$, melanoma (6.0\%), bladder $(6.0 \%)$, thyroid $(6.0 \%)$, kidney $(4.0 \%)$, pituitary tumor $(2.0 \%)$, skin epidermoid carcinoma $(2.0 \%)$, head and neck $(2.0 \%)$, gastric $(2.0 \%)$, cervical $(2.0 \%)$ and not otherwise specified (24.0\%) (data not shown). Twenty percent of the tumors were synchronous and $80.0 \%$ were metachronous. Non-endocrine neoplasms had been previously diagnosed in $70.0 \% \%$ of the patients. Little information is available in the literature regarding the true incidence of second neoplasms in patients with NET (13-15). The association rate was $11.0 \%$ in our series, primarily with NET of the small bowel and pancreas (data not shown).

The overall survival was significantly higher for patients whose tumors were diagnosed at an early stage, were of gastrointestinal origin, or were well-differentiated or low-grade. The prognosis also differed according to tumor type, including non-functioning gastrointestinal, small bowel carcinoid, functioning pancreatic, non-functioning pancreatic and unknown primary tumors (Table VII). The multivariate analysis confirmed stage at diagnosis, tumor subtype, tumor grade and evidence of surgery as independent prognostic factors for survival (Table VIII).

\section{Discussion}

This study provides relevant information regarding the epidemiology, incidence and management of NET in Argentina. Although other countries have their own registries (2-4), we considered it important to use Argentina-specific data in order to achieve a better understanding of the disease in this area and to optimize available diagnostic and therapeutic resources.

We distinguished the different clinical presentations. Abdominal pain was the most frequent symptom (32.0\% of the patients), followed by diarrhea (17.3\% of the patients). Both symptoms are non-specific, which may explain the late diagnosis and advanced disease stage in $59.2 \%$ of the patients. The initial presentation pattern was carcinoid syndrome in $26.0 \%$ of the patients, which was similar to what has been reported in other recent series $(16,17)$. We observed a different biological behavior confirming the heterogeneity of NET. Survival differed based on histological subtype, although the study's total population maintained a 5-year survival rate of $65.1 \%$. Our series demonstrated a distribution similar to that observed in the recently published study of the National Cancer Registry of Spain (Grupo Español de Tumores Neuroendocrinos; GETNE) (17). As expected, the gastrointestinal tract (58.6\%) was the most prevalent site, followed by the pancreas (25.2\%) and primary unknown (16.3\%). The small bowel (26.9\%) was the most frequent location of primary gastrointestinal tumors, followed by the colon-rectum (12.4\%) and appendix (7.6\%). The incidence of colon-rectum reported by the GETNE group (17) was marginally lower (10.5\%) and the other most frequent locations were reported to be the jejunum-ileum, appendix and stomach, which was different from our results. Other series or registries of European countries also indicated the small bowel as the most frequent tumor location (18). An observation that was shared by our study and others $(16,17)$ is the 
presence of disseminated disease at diagnosis $(59.2 \%$ of patients in the present study); however, this may have been biased in our study. In Argentina, patients with disseminated disease at diagnosis are frequently reported and followed up by oncologists; thus, our study may involve a certain extent of underreporting by endocrinologists, gastroenterologists and other specialists. The presence of non-specific symptoms may delay diagnosis; in our series, the most frequently observed non-specific symptom was abdominal pain $(32.0 \%)$. The primary locations most frequently associated with metastasis were the jejunum-ileum (72.9\%), pancreas $(63.8 \%)$ and colon-rectum $(13.9 \%)$. These differ from SEER data, which indicate the pancreas $(64.0 \%)$, colon $(4.0 \%)$ and jejunum-ileum $(30.0 \%)$ as the most frequent sites associated with advanced-stage disease (9).

Patients with poorly differentiated carcinomas exhibited a worse prognosis (18.4\% with 5-year survival) and presented with metastatic disease at the time of diagnosis (57.4\% of the patients). In 457 of the 461 patients, diagnosis was established according to guidelines of the European Consensus through the European Neuroendocrine Tumor Society, following the parameters of the WHO 2000 classification. In 340 of the 457 patients, there was a correlation with the new WHO 2010 classification; to the best of our knowledge, this constituted the first such correlation with patient data in Latin America. After the ARGENTUM work group was created in our country, we noted the high percentage of serum chromogranin A studies (72.8\% of the patients) and the use of octreoscan (gold standard) to adequately stratify patients with NET $(46.5 \%$ of the patients). These data are considered relevant when compared, for example, with data from Spanish investigators (41.0\% for chromogranin A determination and $50.0 \%$ for octreoscan) (17). In 2009, German investigators reported the use of somatostatin receptor scintigraphy (56.0\%) and specific laboratory tests (67.0\%) (19). Conversely, in some Latin American countries, chromogranin A determinations are not used as part of the routine work-up in NET patients. We also observed the application of immunohistochemistry, with the study of at least two NET markers, such as synaptophysin and chromogranin, in tumor tissues (79.9 and 85.6\%, respectively). Ki-67 studies were conducted in $73.7 \%$ of patients, comparing favorably with the GETNE data (17), which reported $36.0 \%$ for this prognostic factor.

We observed a rate of $10.6 \%$ of incidental diagnosis vs. $22.0 \%$ in the GETNE group. The diagnostic yield with incisional or excisional biopsy was high. Diagnoses were reached in $89.7 \%$ of the patients, with immunohistochemistry techniques performed in the samples obtained.

We made extensive use of systemic treatment, such as somatostatin analogues $(30.3 \%)$ and surgery with curative or palliative intent $(67.32 \%)$ and less frequent use of locoregional ablative approaches, such as regional procedures $(8.7 \%)$ and radionuclide therapy $(1.7 \%)$, indicating the different practices performed in different centers and the deficit in teams able to perform these types of procedures.

The 5-year survival rate of $65.1 \%$ in the total population was considered favorable. As reported in other series, we observed significant differences in this measure between gastrointestinal and pancreatic tumors (74.0 vs. 56.3\%; $\mathrm{P}=0.011$ ). Survival was significantly higher in early disease stages and low-grade tumors. The prognosis also differed significantly according to tumor type, primary tumor site, $\mathrm{Ki}-67$ and grading tumor classification. In the multivariate analysis model, we identified stage at diagnosis, tumor subtype, tumor grade and surgery as independent outcome predictors.

In conclusion, we described diverse aspects associated with the treatment of patients with NET and the use of different treatment strategies, diagnostic procedures and follow-up modalities by our work group, ARGENTUM. However, despite the recent improvements, delayed diagnosis remains high in NET patients. To the best of our knowldge, this study constitutes the first account of NET patients in our region with adequate follow-up and survival data.

\section{Acknowledgements}

Funding for this study was provided by Novartis Argentina. We would like to thank Lucila Lavigna and all the physicians who assisted with this project. We would also like to thank Maria Monneret de Villars, who provided medical writing assistance. We particularly thank Dr Gianfranco Delle Fave for his invaluable support with this project.

\section{References}

1. Newton JN, Swerdlow AJ, dos Santos Silva IM, Vessey MP, Grahame-Smith DG, Primatesta P and Reynolds DJ: The epidemiology of carcinoid tumours in England and Scotland. Br J Cancer 70: 939-942, 1994.

2. Janson ET, Holmberg L, Stridsberg M, Eriksson B, Theodorsson E, Wilander E and Oberg K: Carcinoid tumors: analysis of prognostic factors and survival in 301 patients from a referral center. Ann Oncol 8: 685-690, 1997.

3. Levi F, Te VC, Randimbison L, Rindi G and La Vecchia C: Epidemiology of carcinoid neoplasms in Vaud, Switzerland, 1974-97. Br J Cancer 83: 952-955, 2000.

4. Quaedvlieg PF, Visser O, Lamers CB, Janssen-Heijen ML and Taal BG: Epidemiology and survival in patients with carcinoid disease in The Netherlands. An epidemiological study with 2391 patients. Ann Oncol 12: 1295-1300, 2001.

5. Modlin IM, Lye KD and Kidd M: A 5-decade analysis of 13,715 carcinoid tumors. Cancer 97: 934-959, 2003.

6. Kloppel G, Perren A and Heitz PU: The gastroenteropancreatic neuroendocrine cell system and its tumors: the WHO classification. Ann NY Acad Sci 1014: 13-27, 2004.

7. Yao JC, Hassan M, Phan A, Dagohoy C, Leary C, Mares JE, Abdalla EK, Fleming JB, Vauthey JN, Rashid A and Evans DB: One hundred years after 'carcinoid': epidemiology of and prognostic factors for neuroendocrine tumors in 35,825 cases in the United States. J Clin Oncol 26: 3063-3072, 2008.

8. Modlin IM, Oberg K, Chung DC, et al: Gastroenteropancreatic neuroendocrine tumours. Lancet Oncol 9: 61-72, 2008.

9. Öberg K: What can be achieved in NET management today? Presented at 5th Annual ENETS Conference for The diagnosis and treatment of neuroendocrine tumor disease, March 6-8, 2008, Paris, France.

10. Costa F, Domenichini E, Garavito G, Medrano R, Mendez G, O'Connor J, Rojas W, Torres S, Younes RN, Delle Fave G and Oberg K: Management of neuroendocrine tumors: a meeting of experts from Latin America. Neuroendocrinology 88: 235-242, 2008.

11. Klöppel G, Couvelard A, Perren A, et al; Mallorca Consensus Conference participants; European Neuroendocrine Tumor Society: ENETS Consensus Guidelines for the Standards of Care in Neuroendocrine Tumors: towards a standardized approach to the diagnosis of gastroenteropancreatic neuroendocrine tumors and their prognostic stratification. Neuroendocrinology 90: 162-166, 2009.

12. Bosman FT, Carneiro F, Hruban RH and Theise ND (eds): WHO Classification of Tumours of the Digestive System: IARC Press, Lyon, pp 77-82 and 137-139, 2010. 
13. Habal N, Sims C and Bilchik AJ: Gastrointestinal carcinoid tumors and second primary malignancies. J Surg Oncol 75: 310-316, 2000.

14. Tichansky DS, Cagir B, Borrazzo E, Topham A, Palazzo J, Weaver EJ, Lange A and Fry RD: Risk of second cancers in patients with colorectal carcinoids. Dis Colon Rectum 45: 91-97, 2002.

15. Fendrich V, Waldmann J, Bartsch DK, Schlosser K, Rothmund M and Gerdes B: Multiple primary malignancies in patients with sporadic pancreatic endocrine tumors. J Surg Oncol 97: 592-595, 2008.

16. Niederle MB and Niederle B: Diagnosis and treatment of gastroenteropancreatic neuroendocrine tumors: current data on a prospectively collected, retrospectively analyzed clinical multicenter investigation. Oncologist 16: 602-613, 2011.
17. Garcia-Carbonero R, Capdevila J, Crespo-Herrero G, et al: Incidence, patterns of care and prognostic factors for outcome of gastroenteropancreatic neuroendocrine tumors (GEP-NETs): results from the National Cancer Registry of Spain (RGETNE). Ann Oncol 21: 1794-1803, 2010

18. Hauso O, Gustafsson BI, Kidd M, Waldum HL, Drozdov I, Chan AK and Modlin IM: Neuroendocrine tumor epidemiology: contrasting Norway and North America. Cancer 113: 2655-2664, 2008.

19. Ploeckinger U, Kloeppel G, Wiedenmann B, Lohmann R and representatives of 21 German NET centers: The German NET-registry: an audit on the diagnosis and therapy of neuroendocrine tumors. Neuroendocrinology 90: 349-363, 2009. 